\title{
Image Enhancement for In-vivo Breast Cancer Screening using DIET system
}

\author{
Awais Nazir, Muhammad Shahzad Younis, Muhammad Khurram Shahzad
}

\begin{abstract}
Breast cancer is a leading cause of death among women. Conventional screening methods, such as mammography, and ultrasound diagnosis are expensive and have significant limitations. Digital Image Elasto Tomography (DIET) is a new noninvasive breast cancer screening system that has a potential to be a low cost and reliable breast cancer screening tool. It is based on modal analysis of the breast mass, and stereographic 3D image analysis to detect the stiffer abnormal tissues. However, camera sensor noise, especially Gaussian noise is a major source of Optical Flow (OF) error in this approach to tumor detection. This work studies the performance of different conventional filters, including the standard Gaussian filter tool to remove this noise and produce more robust screening results.

A radical approach, Multiple Frame Noise Removal (MFNR) is proposed, for use in this type of medical image processing instead of a Gaussian filter or other typical image noise removal tools. Its a multiple frame noise removal method where Probability Density Function (PDF) of noise is extracted from the multiple images by characterizing the same pixel positions in multiple images. The noise becomes deterministic, and hence easily removed. The proposed algorithm was applied to a data set from 10 phantom breast tests with a prototype DIET system, and 10 in-vivo samples from healthy women. Comparisons were made to an optimal Gaussian filter form that is commonly used. Reductions in OF error using these digitally imaged data sets was used to compare performance.

Refinement of the images for medical applications requires higher PSNR, which was successfully achieved by using MFNR algorithm. In this study, the algorithm was used to improve the imaging results of a DIET system. The conventional wisdom that states that noise removal and detail preservation are contrasting effects is not true when multiple frames are used for noise removal.
\end{abstract}

Index Terms-Breast Cancer Screening, Digital Image Elasto Tomography (DIET), Image Noise Removal, Image Enhancement, Multiple Frame Noise Removal (MFNR)

\section{INTRODUCTION}

A MONG the leading causes of deaths in women is the Breast Cancer, with 400,000 preventable deaths each year [1]. If detected early, about $95 \%$ of the cases can be cured preferably before the tumor becomes greater than $20 \mathrm{~mm}$ in size. Thereafter, it metastasizes, and the cancer spreads, reducing the survival rate to about $20 \%$. Thus, early detection is critical, and higher screening detection rates are desirable, given the current level is less than $75 \%$ in standard mammography. [1], [2] Presently, Clinical Breast Exam (CBE) or palpation and the Mammography are the primary choices available to detect breast cancer at an early stage. CBE is

Awais Nazir, Muhammad Shahzad Younis, and Muhammad Khurram Shahzad are with National University of Science and Technology, Islamabad 46000, Pakistan. email: \{awais.nazir.ch@gmail.com; muhammad.shahzad@seecs.edu.pk; khuram.toor@gmail.com $\}$

Manuscript received April 19, 2005; revised August 26, 2019.

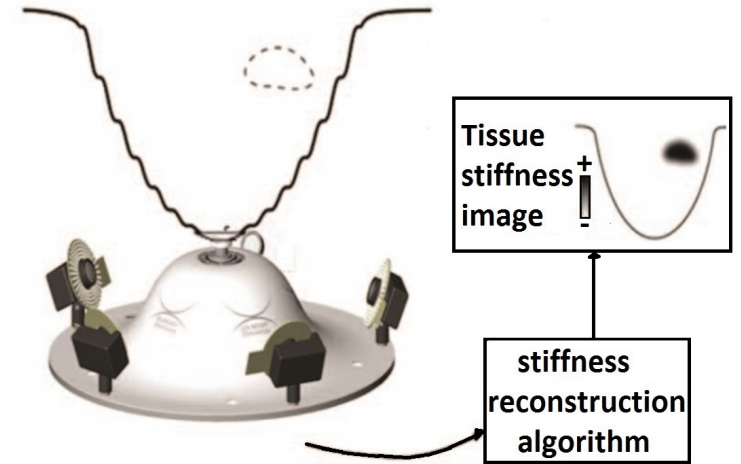

(a)

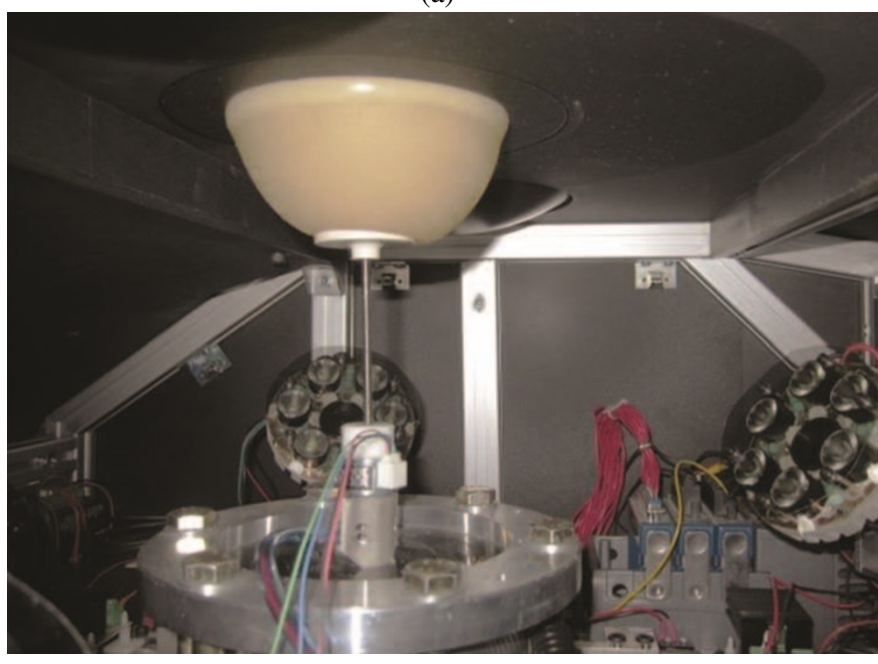

(b)

Fig. 1: DIET System [Digital-Image Elasto-tomography] (a) Vibration at nipple area and image capture. (b) Actual Hardware system.

entirely subjective and Mammography has poor sensitivity because radio-density difference between healthy and cancerous tissues are only 5-10\% [3] putting great stress on the quality of the radiographer analyzing the image. Moreover, mammography requires painful breast compression which reduces compliance. Finally, the subject is prone to accumulated radiation exposure, limiting the eligible age range, and the cost is relatively high. [4], [5] Digital Image Elasto Tomography (DIET) is a new approach. It works on the principle that healthy tissues are 300-1500\% more compliant (less stiff) than their cancerous counterparts. [6] Previous in-vitro results have shown that DIET system is able to detect $10 \mathrm{~mm}$ tumor in 
most of the cases, however, detection of $5 \mathrm{~mm}$ tumors remained inconclusive. [7] A DIET system is fully automated, noninvasive, portable, and thus offers potential for use in younger women with denser breasts, where the diagnostic accuracy of mammography is comparatively less. [8] DIET relies on breast actuation, capturing the breast surface motion using an array of digital cameras, and consequent image processing for diagnostic purposes. [9]-[11] A Clinical prototype is already in use with promising results. [7], [10], [12], [13] Fig. 1 shows overall schematic of the DIET system and use. An actuator induces a sinusoid motion at the nipple area, and 5 cameras are used to create 3D stereo-images with at least 10 phases with respect to the actuator motion. Modal analysis is one possible diagnostic metric [7], [14] along with direct image processing to note how tumors show less phase difference than normal areas due to the elasticity to detect stiffer tumors. [12], [14] Once a tumor is identified, Finite Element Analysis (FEM) is used to detect the exact 3D position of the tumor. [15] A significant problem in DIET cancer detection is the presence of gaussian noise due to low light conditions. [9] The Optical Flow error depended on the ability to process low-noise images. Thus, noise removal was necessary. [14] In traditional software noise removal methods, there is a trade-off between detail preservation and noise removal. Our target is particularly to detect smaller tumors to diagnose early to save lives, thus multiple frames were used to achieve both detail preservation and noise removal. We used multiple frames MFNR method instead of "optimal" Gaussian Filter [14] with excellent results. Other filters which are "standard" at the removal of Gaussian Noise are NLM, Median, Wiener, etc. but they are computationally heavy and require noise PDF before hand while still giving little improvements as shown in the results. In this study, Gaussian, Non-local mean (NLM), Median, Wiener, Kalman Filters, are compared with the proposed MFNR algorithm for the DIET application. The MFNR algorithm is simple to implement and does not need to tune parameters. It is computationally less expensive and scalable to any desired error level.

\section{RELATED WORK}

Multiple frame noise removal methods are only specialized noise removal methods and are not generalized methods. [6] For example, in astronomy, the multiple frames are used to remove the gaussian noise. [6] This paper generalizes the idea of multiple frames to all noise types of all dimensions. We will be comparing the MFNR Algorithm against many standard noise removal Algorithm. Image noise removal is taken as a specific niche case of noise removal. MFNR Algorithm can be applied for noise removal in all types of cases including audio noise removal, energy forcast in powe systems, etc. We will be comparing MFNR Algorithma gainst NLM, Median, Gaussian, Wiener filters at removing noise from colored images.

NLM is one of the "standard" algorithms at removing Gaussian noise from the images. It is much more effective than other noise removal tools for the removal of the Gaussian Noise. [16] There exist other non-local means algorithms which takes only the immediate surround pixel values but

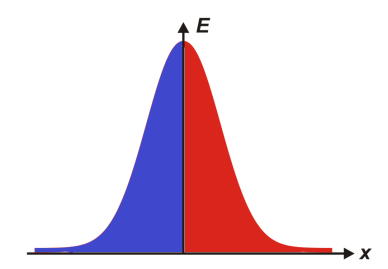

(a)

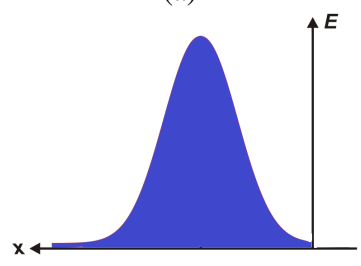

(c)

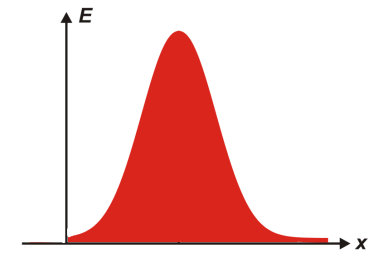

(b)

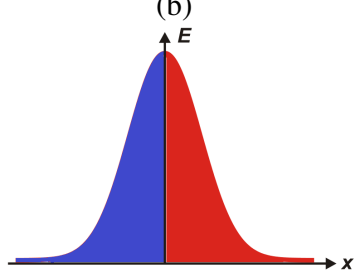

(d)
Fig. 2: Four types of Noise PDFs. (a) Centered Noise. (b) Positive Noise. (c) Negative Noise. (d) Mixed Noise

NLM takes into account all the pictures in the image thus have greater detail preservation results. [17] Problem with NLM though, is that it leaves the "white noise" in the process more like "method noise" or notorious background noise which harms the image details. [18] The MFNR doesn't leave any background noise because it is not smoothing or bull-dozing the feature details unlike "conventional noise removal tools". In short, MFNR Algorithm can replace NLM in image noise removal, [19] image and data processing, [20] interpolation, and depth map regularization and various data processes e.g. interpolation. [19]

Similarly, Median Filter is also compared against MFNR Algorithm. [25] NLM is not good at preserving edges, but Median filters are good at it. Edge detection is required in Optical Flow error to improve the DIET system and in many other applications. Median filters take the median value of the surrounding pixels and replace the pixel value by that median value. Basically, through a "sliding window" action, all the pixels are scanned and the the image "cleaned". It can work on multiple dimentional data also, e.g. on audio data, greyscale images, colored images or video.

\section{Proposed Method}

In multiple frame images, the multiple frames can be used to extract the features of the noise, hence its characteristic or PDF. This PDF is "deterministic" and hence it can be removed completely to output a completely clean image. This is not the case in single frame images where we can't extract the noise PDF characteristics.

When an image is taken, noise is added to it. Depending on the application, it can be gaussian, Poisson, logarithmic, gamma noise, etc. We can extract the features of the noise through multiple frames, and make it deterministic and hence remove it. More the number of frames used, more accurately we can characterize the PDF of the noise and more accurately we can remove it. The multiple frames are taken at the same resolution and position, with the same alignment.

Currently, only niche applications use multiple noise removal systems, like the astronomy project which uses multiple 


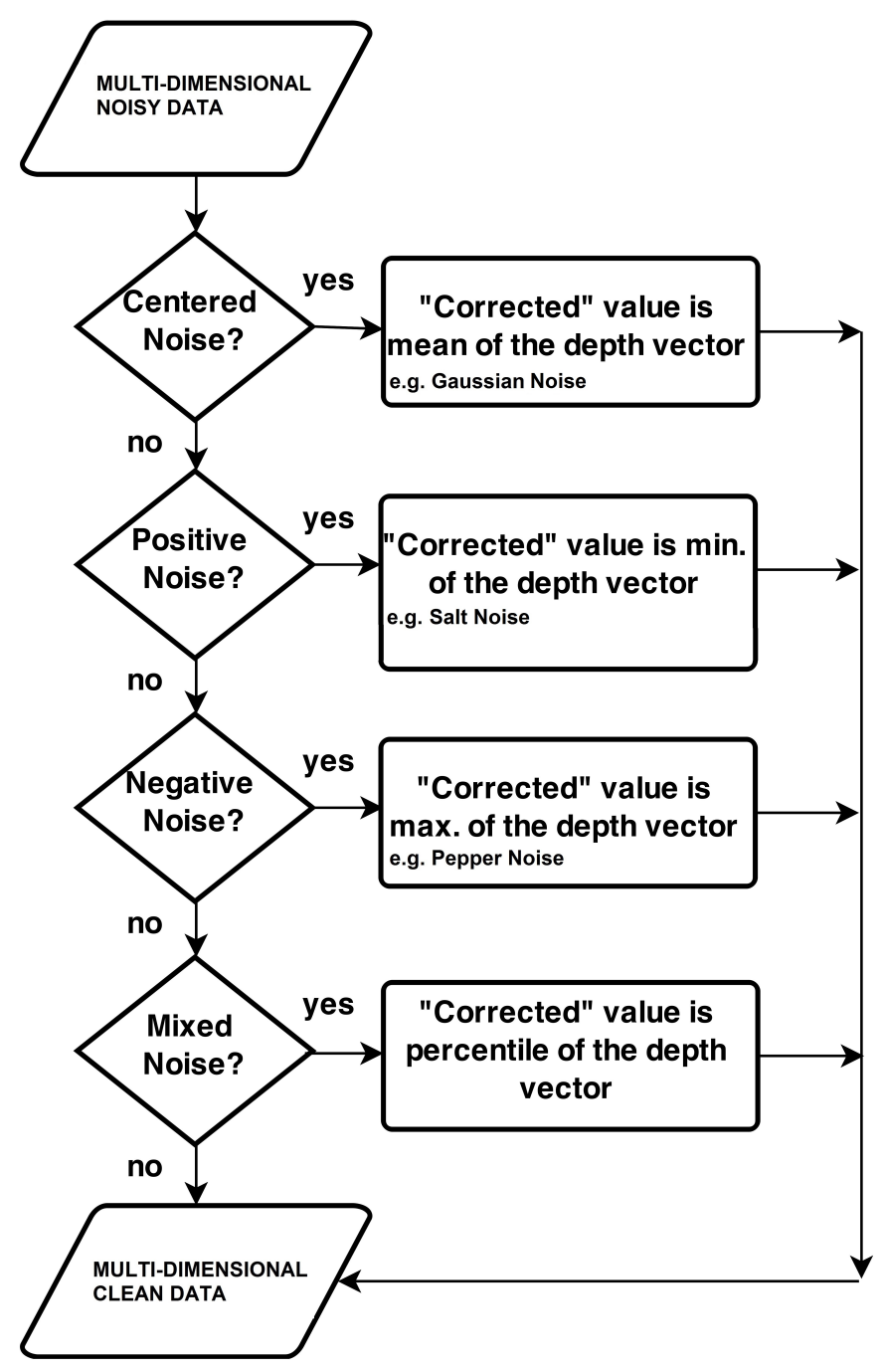

Fig. 3: MFNR Algorithm [Multiple-Frame Noise Removal

images to remove the gaussian noise in its cameras which is prominent in low-light conditions. Each pixel can comprise of whole galaxies so its significant to remove the noise completely. We generalized this multiple frame noise removal system for all noise types and all dimensions.

There are four Noise PDF types: Centered, Positive, Negative, Mixed as shown in Fig. 2. Centered noise has the mean zero for the noise e.g. Gaussian Noise. The Positive Noise has noise added in positive direction e.g. Salt Noise. the Negative Noise has noise added in the negative direction e.g. Pepper Noise. Mixed noise has a mean which is not centered, it maybe more in the positive or negative direction and hence need to be calculted through KDE Algorithm or can be approximated through Centered Noise. Mixed Noise can be examplified by Gamma, Rayleigh, Poisson, Exponential Noise.

The type of noise needs to be known beforehand, e.g. the gaussian noise has a unique signature which can be removed by taking mean of the multiple images. Herein in the DIET system we will be removing the Gaussian noise. But in more complex types of noises like mixed noises, e.g. Poisson or Gamma noise in RADAR applications, we need to use KDE to estimate the mean of the noise and hence remove it. KDE

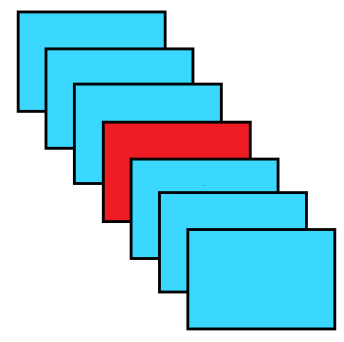

(a)

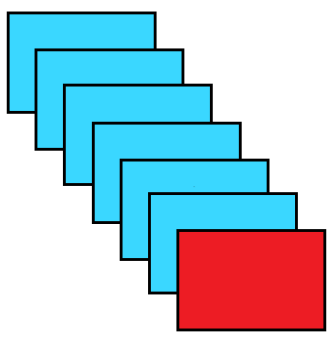

(c)

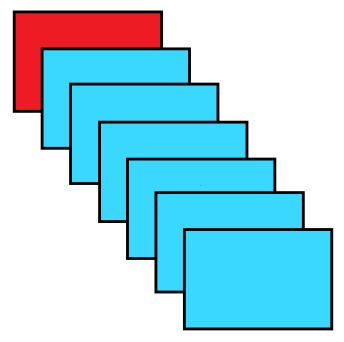

(b)

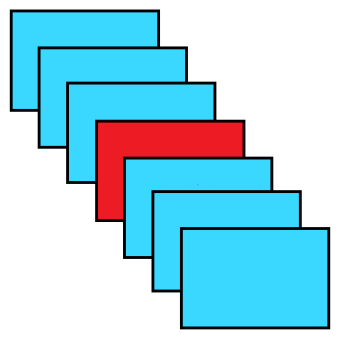

(d)
Fig. 4: Four types of Noise PDFs and their noise cleaning Method. (a) Mean for Centered Noise. (b) Min for Positive Noise. (c) Max for Negative Noise. (d) Kernel Density Estimation (KDE) Mean for Mixed Noise

can be approximated by mean if it fits the bill. General scheme is described in Fig. 3. Multiple images are fed, and one clean image is output. We require the type of noise to remove it.

The multiple frame are assembled such as to make a $3 \mathrm{D}$ stack. The "depth vector" is defined as the pixel value for the same position in each frame. This "depth vector" helps determine the noise PDF and hence remove it in the clean image. Fig. 4 shows the process for each noise type.

\section{EXPERIMENTAL SETUP}

The results were generated using Jupyter running Python on Windows x64 system. All the noise was simulated using standard libraries or otherwise explicitly mentioned.

The sequence of operations in DIET system are as shown in Fig. 1. Fig. 1 (a) shows Camera calibration for 3D stereo vision [21]. Fig. 1 (b) shows segment breast imaging, i.e. vibrating breast at the nipple through actuator and taking 10 pictures for each phase of the actuator through 5 cameras, totaling 50 images for each cycle. This is repeated for all frequencies of interest normally from $10 \mathrm{~Hz}$ to $80 \mathrm{~Hz}$. The resulting images are intensity homogenized because optical flow is very sensitive to non-homogenized intensity images resulting in original system.

Surface 3D reconstruction is done through silhouette method through least squares fitting.

A Horn-Schunck optical flow method is used to calculate optical flow in 3D, [22] which in turn is used to identify skin surface points' phase with respect to the actuator.

First and Second natural frequencies are identified. Phase detection near the chest and nipples are ignored because boundary conditions dominate here. 10 Modal Analysis is used to detect the cancerous tissue(s) location. [7], [14] 


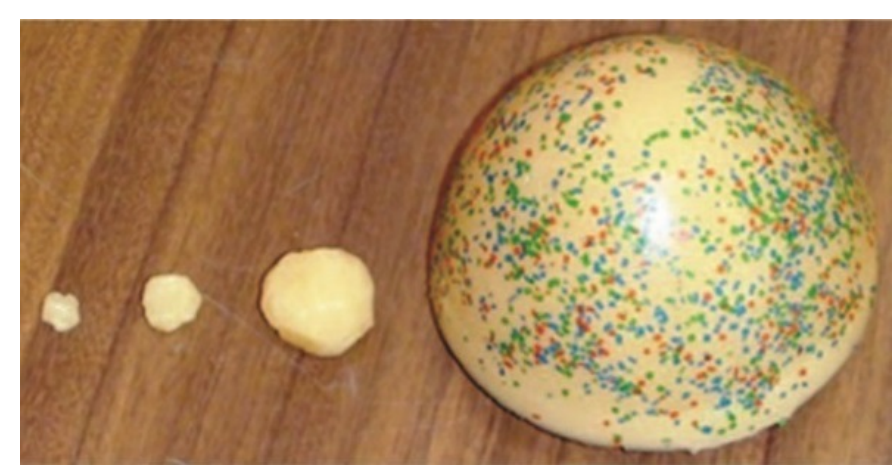

(a)

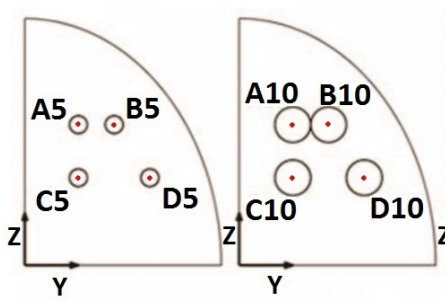

(b)

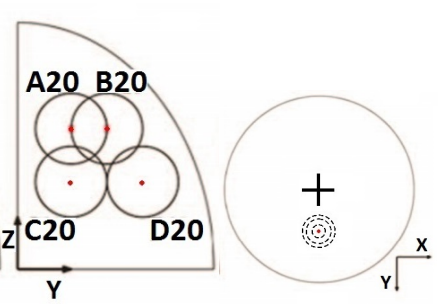

(c)
Fig. 5: DIET Phantoms (a) Phantom and tumors of 20mm, $10 \mathrm{~mm}$, and $5 \mathrm{~mm}$. (b) Location of each tumor and its size e.g. A5 means Position A with $5 \mathrm{~mm}$ tumor. (c) Location of tumors from front.

To make a reference system for different tumor diameters at different locations, and to do ex-vivo trials before moving to human trials, silicone breast phantoms were designed using different silicone materials [23], [24] as shown in Fig. 5. The tumors were from stiffer material mimicking tumor elasticity, and breast mass from flexible material mimicking real breast elasticity. The surface of the phantoms was coated with stiffer material mimicking human skin. Phantoms were necessary for repeatable experiments and allow more perfect repeated experiments. Fig. 5 shows the tumor locations and respective sizes at each location.

SNR and Optical Flow Error calculations were carried out comparing the performance of various filters including Gaussian, NLM, Median, Wiener, and the proposed MFNR algorithm. Gaussian noise removal in simulation, phantoms, and in-vivo images in DIET Project were done using Jupyter/Python and the results compared.

More specifically, the relative OF errors were evaluated first on data simulated by warping real images, so that absolute ground truth is available, then the same procedure is replicated for phantom and in-vivo data. To simulate sensor noise, Gaussian noise with standard deviation of 5 grayscale levels was added to each pixel for each color channel. [14]

\section{Performance Evaluation}

\section{A. Performance Measurements}

PSNR was calculated for the images to compare the performance of the different noise removal algorithms including MFNR. PSNR is depicted as in Eq. 1.

$$
\operatorname{PSNR}=10 \log _{10} \frac{\left(2^{d}-1\right)^{2} W H}{\sum_{i=1}^{W} \sum_{j=1}^{H}\left(p[i, j]-p^{\prime}[i, j]\right)^{2}}
$$

Secondly, Optical Flow (OF) Error was also calculated as a performance measure. We required OF Error to be reduced to accurately identify the $5 \mathrm{~mm}$ tumors. Optical Flow basically determines how much features can be detected between two successive frames after they have undergone motion. Thus, we are considering a sequence of images $\left(I(t)_{\{t=1 \cdots T\}}\right)$ taken from the motion between frames. Pixels are having a geometrical transformation between the two frames. We are trying to find out the path of each pixel $\mathbf{x}_{0}(t)=\left(x_{0}(t), y_{0}(t)\right)$ from its start position $\mathbf{x}_{0}$. It can be mathematically formulated as in Eq. 2.

$$
I\left(\mathbf{x}_{0}(t), t\right)=I\left(\mathbf{x}_{0}, 0\right)
$$

Its derivative begets Eqn. 3

$$
0=\frac{d}{d t} I\left(x_{0}(t), t\right)=\nabla I . u+I_{t}
$$

where $\mathbf{u}=\left(u_{x}, u_{y}\right)=\frac{d \mathbf{x}_{0}}{d t}=d \mathbf{x}_{0}$ is the motion of the pixel. Let it be noted that Eq. 3 is insufficient to find $\mathbf{u}$ (our target).

This is an optimization problem and we need to find Eq. 4.

$$
E(\mathbf{u})=\sum_{\mathbf{x}} g_{\mathbf{x}_{0}}(\mathbf{x})\left[\nabla I(\mathbf{x}, t) \cdot \mathbf{u}+I_{t}(\mathbf{x}, t)\right]^{2}(\mathbf{x})
$$

(Here $g_{\mathbf{x}_{0}}$ is a Gaussian filter centered at $\mathbf{x}_{0}$ ).

When the gradiant of $E$ vanishes, we achieve the minumum as in 5 and 6

$$
\begin{aligned}
& \frac{\partial E\left(u_{x}, u_{y}\right)}{\partial u_{x}}=\sum_{\mathbf{x}} g_{\mathbf{x}_{0}}(\mathbf{x})\left[u_{x} I_{x}^{2}+u_{y} I_{x} I_{y}+I_{x} I_{t}\right](\mathbf{x})=0 \\
& \frac{\partial E\left(u_{x}, u_{y}\right)}{\partial u_{y}}=\sum_{\mathbf{x}} g_{\mathbf{x}_{0}}(\mathbf{x})\left[u_{y} I_{y}^{2}+u_{x} I_{x} I_{y}+I_{y} I_{t}\right](\mathbf{x})=0
\end{aligned}
$$

where, $I_{x}, I_{y}$ and $I_{t}$ represent respectively the derivative of the image sequence relatively to $x, y$ and $t$. This linear system can be expressed as in Eq. 7.

$$
M_{\mathbf{x}_{0}} \mathbf{u}=b_{\mathbf{x}_{0}}
$$

where,

$$
\begin{aligned}
M_{\mathbf{x}_{0}} & =\left(\begin{array}{cc}
\sum g_{\mathbf{x}_{0}} I_{x}^{2} & \sum g_{\mathbf{x}_{0}} I_{x} I_{y} \\
\sum g_{\mathbf{x}_{0}} I_{x} I_{y} & \sum g_{\mathbf{x}_{0}} I_{y}^{2}
\end{array}\right) \\
b_{\mathbf{x}_{0}} & =-\left(\begin{array}{c}
\sum g_{\mathbf{x}_{0}} I_{x} I_{t} \\
\sum g_{\mathbf{x}_{0}} I_{y} I_{t}
\end{array}\right)
\end{aligned}
$$

\section{B. Performance Analysis}

Multiple images are required for the MFNR algorithm, and the larger the number of images, the better the noise removal. The number of images required depends on the accuracy to be achieved. However, the DIET concept takes several such images as part of its normal approach, which is thus a good match between the filter and the DIET system requirements. The algorithm has numerous features making it preferable 


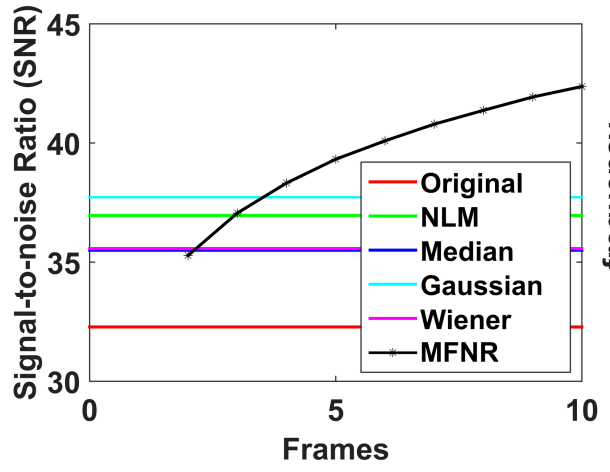

(a)

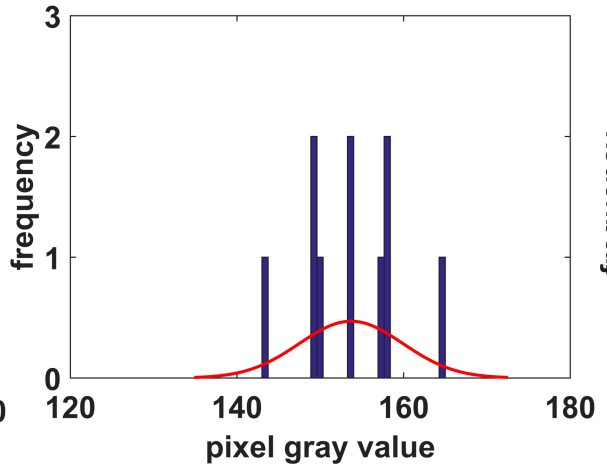

(b)

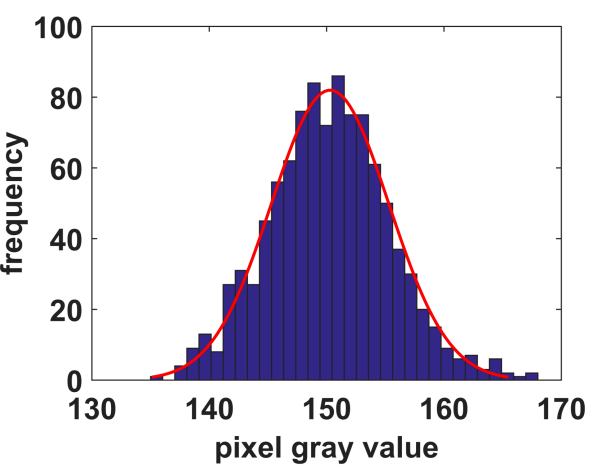

(c)

Fig. 6: (a) SNR comparison on simulated noise images. (b) "depth vector" for 10 images. True pixel value is 150 , corrected value is 151.9. (c) "depth vector" for 1000 images. True pixel value is 150 , corrected value is 150.1 .

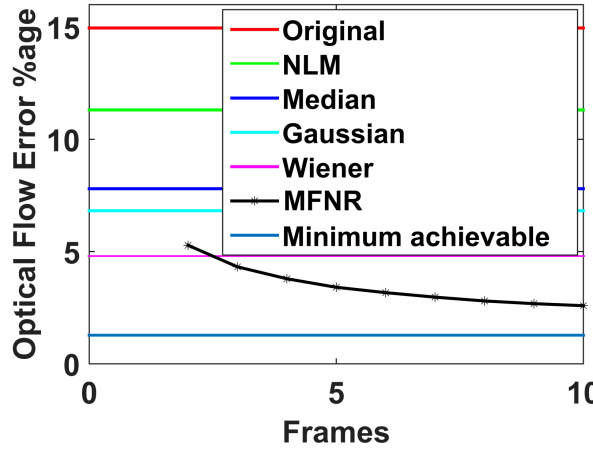

(a)

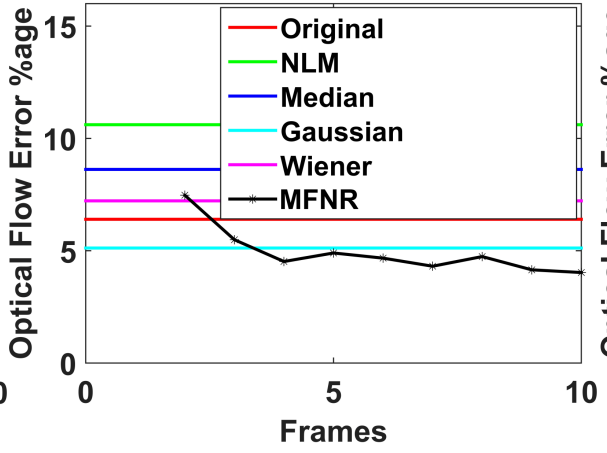

(b)

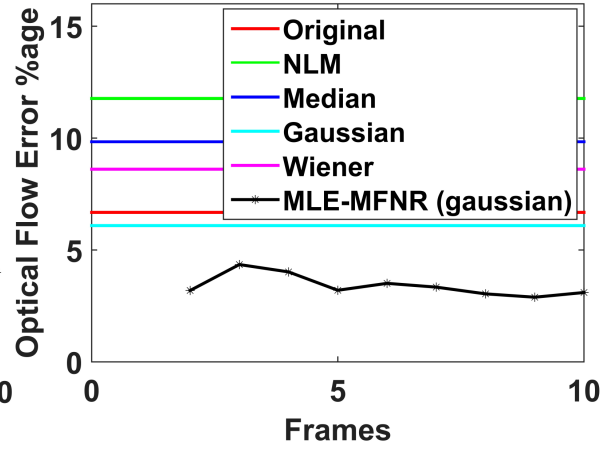

(c)

Fig. 7: Optical Flow Error Percentage on (a) Simulated noisy images (b) 10 phantom images (c) in-vivo images of 10 human subjects

over other noise removal tools. While using conventional noise removal algorithms, their parameters are often unknown most of the time, and frequently need to be estimated. For example, for applying NLM filter, patch size, window size, and noise level has to be given. The results show that the proposed filter is simpler, and performs better than more optimal filters like Gaussian, or Wiener Filter, and other complex filters.

Multiple images can be taken through high frequency video cameras as a video. Then those video can be converted into multiple frames given the time delay is negligible. The storage and computational requirements are much less than similar algorithms. Generally the MFNR Algorithm computational requirements are modest and can be implemented on standard equipment without the need of any specialized equipment.

In DIET application, comparison was made between the simulated, phantom and in-vivo images. MFNR algorithm gave variable results when applied to phantom and in-vivo images than its "default" performance using simulated pairs. Still, it reduced error significantly compared to the "optimal" Gaussian filter used by Botterill et al. [14] in the previous best result. Two images from the MLE-MFNR algorithm successfully met requirements, nearly halving the OF error from the previous best result. The results were variable because the OF error was highly sensitive to slight unwanted camera vibrations and luminosity difference between frames, both of which can be present in images from a DIET system. In this paper, MFNR Algorithm improved upon DIET system data. However, MFNR Algorithm can remove most kind of noise given their PDF characteristics. In fields like medicinal image processing, RADAR, cameras, astronomy, MFNR offer a potentially better way to remove noise. For example, in low light conditions, cameras have a significant gaussian noise which makes computer visibility an undauntable task especially in critical applications like Autonomous Vehicles. MFNR Algorithm can fix that and allow noise-free visibility for Autonomous Vehicles even during night. MFNR Algorithm can also remove periodic noise like in Energy Forecast prediction based on past data, given the past data have noise which can be determined through calculating its PDF.

\section{Performance Results}

Eq. 10 shows the Gaussian noise added to our raw images, where $\mu$ shows mean of the Gaussian noise distribution which is 0 , and $\sigma$ is the standard deviation of the Gaussian distribution.

$$
p(x)=\frac{1}{\sigma \sqrt{2 \pi}} e^{\frac{-(x-\mu)^{2}}{2 \sigma^{2}}}
$$

Fig. 6 shows the image noise removal comparison between different filters using simulated noise. A skin surface was 
taken that was typically encountered in the DIET system and Gaussian noise added. Previously, Gaussian filters gave the maximum performance, but the MFNR Algorithm showed far greater PSNR performance. It was estimated that as the number of images were increased, the noise was removed in an exponential manner, improving in ways the other filters could not. The accuracy can be scaled according to requirements. Fig. 7 shows the average Optical Flow Error performance using simulated, in-vivo images from 10 "human subjects", and 10 phantom images. Optical Flow Error graph is haphazard but general trend is downwards. It is haphazard because it is based on Optical Flow algorithm which is trying to match feature between two successive frames, so it depends on the accuracy of the Optical Flow Algoritm.

\section{CONCLUSION}

The proposed MFNR algorithm was found to perform much better at Gaussian noise removal in the DIET system than conventional noise removal tools. Optical Flow error was halved using proposed MFNR Algorithm compared to using more optimal Gaussian filters as in prior work. [14] Improved PSNR and measurement precision will improve the resulting screening capability. The overall MFNR algorithm is readily generalized to other applications and can remove any type of "deterministic" or known noise profile, and is only limited by this needed knowledge and the need for multiple images. It could thus improve upon many existing systems used to analyze images in medical, astronomy or defense related fields.

\section{REFERENCES}

[1] S.S. Coughlin, D.U. Ekwueme, "Breast cancer as a global health concern," Cancer epidemiology 33, 315-318 (2009).

[2] M.o.H. New Zealand Health Information Services (NZHIS), Wellington, "Cancer Patient Survival Covering the Period 1994 to 2003," 2006).

[3] D. Kopans, "Breast anatomy and basic histology, physiology, and pathology," Kopans DB. Breast Imaging, 3rd Edition. Philadelphia: Lippincott Williams and Williams, 7-43 (2007).

[4] P.C. Gøtzsche, K.J. Jørgensen, P.-H. Zahl, J. Mæhlen, ”Why mammography screening has not lived up to expectations from the randomised trials," Cancer Causes \& Control 23, 15-21 (2012).

[5] A. Sarvazyan, V. Egorov, J. Son, C. Kaufman, "Cost-effective screening for breast cancer worldwide: current state and future directions," Breast cancer: basic and clinical research 1, 91 (2008).

[6] A. Samani, J. Zubovits, D. Plewes, "Elastic moduli of normal and pathological human breast tissues: an inversion-technique-based investigation of 169 samples," Physics in medicine and biology 52, 1565 (2007).

[7] A.S. Kashif, T.F. Lotz, A.M. Heeren, J.G. Chase, "Separate modal analysis for tumor detection with a digital image elasto tomography (DIET) breast cancer screening system," Medical physics 40, 113503 (2013).

[8] W.A. Berg, L. Gutierrez, M.S. NessAiver, W.B. Carter, M. Bhargavan, R.S. Lewis, O.B. Ioffe, "Diagnostic accuracy of mammography, clinical examination, US, and MR imaging in preoperative assessment of breast cancer 1," Radiology 233, 830-849 (2004).

[9] R.G. Brown, J.G. Chase, C.E. Hann, "A pointwise smooth surface stereo reconstruction algorithm without correspondences," Image and Vision Computing 30, 619-629 (2012).

[10] T. Lotz, A. Kashif, S. Feng, P. Biret, Y. Denais, D. Lottin, L. Maillard, T. Tirschler, J.G. Chase, presented at the Bioinformatics and Biomedical Engineering,(iCBBE) 2011 5th International Conference on 2011.

[11] E.E. Van Houten, H. Kershaw, T. Lotz, J.G. Chase, presented at the Engineering in Medicine and Biology Society (EMBC), 2012 Annual International Conference of the IEEE 2012.
[12] T. Lotz, P.D. Simpson, D. Stocker, C.E. Hann, J.G. Chase, presented at the IEEE EMBS 2010.

[13] A.S. Kashif, Richard Wien (Ed.), Digital Image Elasto Tomography (DIET) Breast Cancer Screening System. (ISBN: 978-3-639-51259-5; Scholar's Press, Saarbrucken, Germany, 2015).

[14] T. Botterill, T. Lotz, A. Kashif, J.G. Chase, "Reconstructing 3-D Skin Surface Motion for the DIET Breast Cancer Screening System," IEEE Trans. Med. Imaging 33, 1109-1118 (2014).

[15] H.-U. Berger, C.E. Hann, J.G. Chase, R.L. Broughton, E. Van Houten, presented at the Medical Imaging 2007.

[16] Coupé, Pierrick, et al. "An optimized blockwise nonlocal means denoising filter for 3-D magnetic resonance images." IEEE transactions on medical imaging 27.4 (2008): 425-441.

[17] Kervrann, Charles, Jérôme Boulanger, and Pierrick Coupé. "Bayesian non-local means filter, image redundancy and adaptive dictionaries for noise removal.” International conference on scale space and variational methods in computer vision. Springer, Berlin, Heidelberg, 2007.

[18] Kumar, BK Shreyamsha. "Image denoising based on non-local means filter and its method noise thresholding." Signal, image and video processing 7.6 (2013): 1211-1227.

[19] A. Buades, Y. Lou, J.-M. Morel, Z. Tang, "Multi image noise estimation and denoising," preprint, Aug 2010).

[20] S.D. Gwyn, "MegaPipe: The MegaCam image stacking pipeline at the Canadian astronomical data centre," Publications of the Astronomical Society of the Pacific 120, 212-223 (2008).

[21] J.G. Chase, C.E. Hann, L.A. Ray, "Global motion invariant signatures for fast and accurate motion tracking in a digital image-based elastotomography system," (Google Patents, 2012).

[22] J.L. Barron, D.J. Fleet, S.S. Beauchemin, "Performance of optical flow techniques," International journal of computer vision 12, 43-77 (1994).

[23] A. Peters, J.G. Chase, E.E. Van Houten, "Digital image elastotomography: mechanical property estimation of silicone phantoms," Medical \& biological engineering \& computing 46, 205-212 (2008).

[24] R.G. Brown, "Three-dimensional motion capture for the DIET breast cancer imaging system," 2008).

[25] Brownrigg, David RK. "The weighted median filter." Communications of the ACM 27.8 (1984): 807-818.

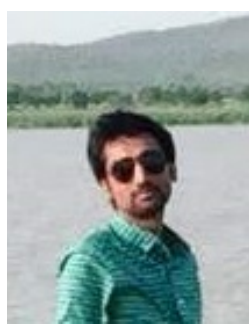

Awais Nazir received his Bachelor's degree in Electrical Engineering from National University of Sciences and Technology (Islamabad, Pakistan) in 2012, and Master's degree from National University of Sciences and Technology in Electrical Engineering in 2015. He has diverse industrial and academia experience in his field as Project Manager at various Engineering and Research Projects. Currently he is pursuing his $\mathrm{PhD}$ in Electrical Engineering from $\mathrm{Na}$ tional University of Sciences. His research interests include Data Science, Artificial Intelligence, BioMedical Engineering, and Computer Vision.

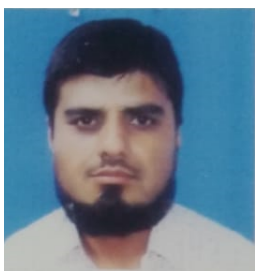

Muhammad Shahzad Younis received the bachelor's degree from National University of Sciences and Technology, Islamabad, Pakistan, in 2002, the master's degree from the University of Engineering and Technology, Taxila, Pakistan, in 2005, and the $\mathrm{PhD}$ degree from University Technology PETRONAS, Perak, Malaysia in 2009, respectively. Before joining National University of Sciences and Technical (NUST), he was Assistant Manager at a research and development organization named AERO where he worked on different signal processing and embedded system design applications. He is currently working as an Assistant professor in the Department of Electrical Engineering in School of Electrical Engineering and Computer Science (SEECS)-NUST. He has published more than 25 papers in domestic and international journals and conferences. His research interests include Statistical Signal Processing, Adaptive Filters, Convex Optimization Biomedical signal processing, wireless communication modelling and digital signal processing. 


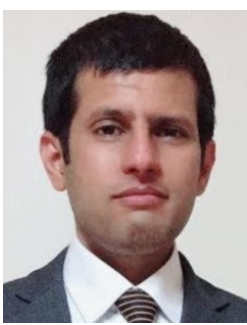

Muhammad Khurram Shahzad received the B.E. and M.S. degrees in Information Technology from The University of Lahore (2004) and National University of Science and Technology (NUST), Islamabad, Pakistan (2007) respectively. $\mathrm{He}$ earned Ph.D. degree in Computer Engineering from Sungkyunkwan University (SKKU), Suwon, South Korea, He joined MONET Lab in SKKU as Postdoctoral Researcher after graduation in 2016.

Later, he worked as Assistant Professor with the Electrical Energy Engineering Department during 2017 to 2018 at Keimyung University, Daegu, South Korea. Currently, he is working as Assistant Professor at Department of Computing, School of Electrical Engineering and Computer Science, NUST, Islamabad, Pakistan. His responsibilities include; PG CS Coordinator, PG Data Science Coordinator, PG CS Interviews, and also worked as QS Ranking Rubrics lead. His research interests include Artificial Intelligence, Data Science, and Graph Theory. 